TRABAJOS ORIGINALES

Rev Obstet Ginecol Venez. 2021; 81 (2): 116-126

https://doi.org/10.51288/00810205

\title{
Síndrome de folículo vacío en mujeres infértiles sometidas a técnicas de reproducción asistida de alta complejidad
}

\author{
D Isolina Marval, ${ }^{1,2}$ (D) Adriana Fernández, ${ }^{2}$ Omar Verde, ${ }^{2}$ Jham Lugo. ${ }^{1}$
}

\begin{abstract}
RESUMEN
Objetivo: Caracterizar el síndrome de folículo vacio en pacientes del Centro de Fertilidad CenFer, en el periodo entre 2013 y 2020. Métodos: Se realizó un estudio retrospectivo, que incluyó 372 ciclos de fertilización in vitro e inyección intracitoplasmática de espermatozoides. Las pacientes fueron sometidas a un estímulo ovárico controlado utilizando antagonistas de la hormona liberadora de gonadotropinas, administrándose hormona gonadotropina coriónica recombinante para desencadenar la maduración de los ovocitos y luego de 36 horas se realizó la aspiración folicular para la obtención de ovocitos. Se incluyeron variables implicadas en los ciclos de fertilización in vitro e inyección intracitoplasmática de espermatozoides antes del aspirado folicular y se consideraron dos grupos: pacientes con sindrome de foliculo vacio y el grupo control.

Resultados: La incidencia del síndrome de folículo vacio fue de 6,18\% y la recurrencia del $20 \%$. La incidencia y recurrencia aumentan significativamente con el incremento de la edad de la paciente $(p<0,05)$. Los casos parecen estar asociados con una baja respuesta ovárica ante la estimulación ovárica controlada ya que, en el grupo de pacientes, la concentración sérica de hormona antimülleriana y del estradiol (día $h C G)$ es significativamente menor $(p<0,05)$ y la concentración sérica de hormona foliculoestimulante es significativamente mayor ( $p<0,05)$; hallazgos asociados al aumento de la incidencia del síndrome de folículo vacio en pacientes de edad avanzada $(p<0,05)$.

Conclusiones: La incidencia y la recurrencia del sindrome de foliculo vacio aumentan con la edad de la paciente. La exclusión o inclusión de las pacientes bajas respondedoras es controversial.
\end{abstract}

Palabras clave: Disminución reserva ovárica, Sindrome foliculo vacio, Fertilización in vitro.

\section{SUMMARY}

Objective: To characterize the empty follicle syndrome present in patients at the fertility center CenFer, between 2013 and 2020. Methods: A retrospective study was carried out. This study included 372 in vitro fertilization and intraovocyte injection of spermatozoa cycles. The patients were subject to a controlled ovarian stimulation using gonadotropin-releasing hormone antagonists, administering recombinant gonadotropin hormone to trigger oocyte maturation and, after 36 hours, the follicular aspiration was performed to obtain oocytes. For this study, variables involved in in vitro fertilization and intraovocyte injection of spermatozoa cycles before follicular aspiration were included and two groups were considered: patients with empty follicle syndrome and the control group.

Results: The incidence of empty follicle syndrome was $6.18 \%$ and recurrence was $20 \%$. The incidence and recurrence increase significantly with increasing age of the patient $(p<0.05)$. The cases seem to be associated with a low ovarian response to controlled ovarian stimulation since in the group of patients the serum concentration of antimüllerian hormone and estradiol $($ day $h C G)$ is significantly lower $(p<0.05)$ and the serum concentration of follicle-stimulating hormone is significantly higher $(p<0.05)$; findings associated with the increased incidence of empty follicle syndrome in elderly patients $(p<0.05)$.

Conclusions: The incidence and recurrence of empty follicle syndrome increase with the patient's age. Exclusion or inclusion of poor responder patients is controversial.

Keywords: Decrease in ovarian reserve, empty follicle syndrome, in vitro fertilization.

\footnotetext{
${ }^{1}$ CenFer, Centro de Fertilidad Clínica Lugo, Maracay, Edo. Aragua. ${ }^{2}$ Universidad Central de Venezuela, Sede Maracay, Edo. Aragua.

Trabajo especial realizado por la Lcda. Isolina Marval para optar al título de Especialista en Reproducción Animal. El trabajo fue distinguido con Mención Honorífica. Parte de este trabajo fue presentado en el Congreso de la Asociación Venezolana de Medicina Reproductiva y Embriología, AVEMRE 2017, en modalidad de Poster.
}

\section{INTRODUCCIÓN}

El síndrome del folículo vacío (SFV) en mujeres, fue descrito por primera vez en 1986 por Coulam y cols. (1), como una condición en la que una vez que se ha realizado una estimulación ovárica controlada con 


\section{SÍNDROME DE FOLÍCULO VACÍO EN MUJERES INFÉRTILES SOMETIDAS A TÉCNICAS DE REPRODUCCIÓN ASISTIDA DE ALTA COMPLEJIDAD}

esteroidogénesis normal y una aspiración folicular meticulosa, no hay recuperación de ovocitos.

La incidencia del SFV es baja, varía entre $0,045 \%$ y $7 \%$ (2); sin embrago, es una complicación frustrante en los procedimientos de fecundación in vitro (FIV), dejando como consecuencia la cancelación del ciclo. Además, puede ser traumático, causando estrés substancial y ansiedad en las pacientes, así como en el personal clínico involucrado en las técnicas de reproducción asistida; por lo tanto, estas implicaciones determinan la importancia del entendimiento del SFV $(3,4)$.

El SFV ha sido clasificado en dos tipos: verdadero o genuino y falso. El primero ha sido definido como una falla en la recuperación de ovocitos a pesar de que las pacientes presenten niveles óptimos ( $>40 \mathrm{mUI} /$ $\mathrm{mL}$ ) de $\beta$-gonadotropina coriónica humana (hCG) el día de la aspiración folicular. El segundo tipo de SFV, ha sido definido como una falla en la recuperación de ovocitos en presencia de un bajo valor de hCG $(<40 \mathrm{mUI} / \mathrm{mL})$ o un valor indetectable el día de la aspiración folicular, debido a un error humano o por razones farmacológicas (5). Entre los errores humanos más comunes cabe mencionar los siguientes: falla al momento de la inyección de hCG, administración de la hormona en el momento no adecuado o inapropiada resuspensión del soluto de hCG en la solución (4).

Según, Stevenson y col. (5), un $33 \%$ de los casos de SFV son clasificados como verdaderos y un $67 \%$ son clasificados como falsos (5), lo que sugiere que la mayoría de los casos de SFV, se presentan por error humano $(3,4)$.

Existen numerosas referencias con relación al SFV, entre ellas, artículos científicos que incluyen estudios observacionales, reportes de casos, reportes de series, revisiones científicas, cartas y recomendaciones (6), aunque no existen reportes en Venezuela que hagan mención del mismo; sin embargo, la etiología del SFV permanece sin dilucidar y su existencia es cuestionada por muchos científicos $(4,7,8)$.

El objetivo de la mayoría de las investigaciones en este tema, radica en manejar exitosamente los ciclos siguientes en las pacientes que han presentado el SFV, realizando cambios en el protocolo de estimulación (3, 4). Otras investigaciones se enfocan en recuperar los ovocitos durante el ciclo en el que se presenta el SFV $(8,9)$ y en algunos reportes, se ha señalado que el SFV es recurrente en las pacientes, sugiriendo un problema adicional a la inadecuada administración de hCG (4, 7). Cuando el SFV es recurrente en una paciente, existe incertidumbre debido a que se ha observado que, incluso, puede persistir en hermanas y en personas con anomalías cromosómicas (4), quedando como única solución la donación de ovocitos (7).

El objetivo de esta investigación fue caracterizar el SFV mediante el análisis de los datos obtenidos en el Centro de Fertilidad de la Clínica Lugo CenFer, en el periodo entre 2013 y 2020.

\section{MÉTODOS}

El estudio se realizó en las instalaciones del Centro de Fertilidad CenFer, ubicado en la Clínica Lugo de la ciudad de Maracay, Edo. Aragua. Se realizó un estudio retrospectivo, donde se incluyeron 372 pacientes que asistieron a CenFer para la realización de un procedimiento de reproducción asistida de alta complejidad: fecundación in vitro (FIV) o inyección intracitoplasmática de espermatozoides (ICSI), entre los años 2013 y 2020.

Para el protocolo de estimulación ovárica controlada (EOC) se utilizaron antagonistas de la hormona liberadora de gonadotropinas $(\mathrm{GnRH})$. Se administraron inyecciones diarias de la hormona folículo estimulante (FSH) recombinante (Gonal F®, 
Merck Serono, Reino Unido; o Puregon ${ }^{\circledR}$, Vetter Pharma Fertigung Gmb H \& Co. KG, Alemania) desde el día 2, 3 o 4 del ciclo, hasta el día de la colocación de la hCG; además, en algunas pacientes, se administró en conjunto hormona luteinizante $(\mathrm{LH})$ recombinante (Luveris ${ }^{\circledR}$, Merck Serono, Reino Unido). La supresión de la adenohipófisis fue llevada a cabo, aproximadamente, a partir del día 9 del ciclo hasta el día anterior al aspirado, mediante la administración de un antagonista de la GnRH (Cetrotide ${ }^{\circledR}$, Merck S.A., Alemania). Posteriormente, se monitoreó el desarrollo folicular como respuesta a la estimulación hormonal, a través de ecografía transvaginal, en conjunto con los niveles séricos de estradiol.

Una vez completado el desarrollo folicular múltiple, es decir, cuando la mayoría de los folículos midió entre 18 y $22 \mathrm{~mm}$, se administró a la paciente 250-500 $\mu \mathrm{g}$ de hCG recombinante (rhCG; Ovidrel ${ }^{\circledR}$, Merck Serono), con la finalidad de imitar el pico preovulatorio endógeno de LH. La aspiración folicular para la obtención de ovocitos se realizó 36 horas posteriores a la administración de rhCG.

El aspirado de los folículos se realizó en el quirófano de FIV mediante aspiración folicular transvaginal, utilizando para ello, una bomba de succión acoplada a un sistema de mangueras que se conecta a la aguja de aspiración; a su vez, la aguja de aspiración se acopla al transductor transvaginal mediante una guía (MySono U6, MEDISON®; Cook Aspiration Unit ${ }^{\mathrm{TM}}$, K-MAR-5200, Cook Medical®; Wallace Oocyte Recovery System, ONS1733750, Wallace $\left.{ }^{\circledR}\right)$. En el monitor se localizaron y visualizaron los folículos, procediéndose a su punción.

En cuanto a la analgesia, se utilizó sedación endovenosa y anestesia local (Propofol y clorhidrato de lidocaína, cifarcaína $1 \%$, Behrens, Venezuela) en el cuello del útero y en el fondo de la vagina.
El sistema de aspiración empleado permitió la recolecta del líquido folicular en los tubos de recolección (14 mL, 35-2057, BD Falcon $\left.{ }^{\circledR}\right)$, los cuales fueron trasladados inmediatamente al laboratorio de FIV, el cual se encuentra adyacente al quirófano, manteniéndose a $37^{\circ} \mathrm{C}$ en el calentador de tubos (Test TubeHeater, K-FTH-1012, Cook Medical®) hasta su evaluación.

El líquido folicular recuperado fue vertido en las placas de búsqueda de ovocitos (placas Petri $90 \mathrm{~mm}$, BioBase ${ }^{\circledR}$ o placas cuadradas $100 \times 15 \mathrm{~mm}$, Gridded, 351,112 , BD Falcon $\left.{ }^{\circledR}\right)$ y mediante la observación a través del microscopio estereoscópico (Olympus ${ }^{\circledR}$, SF10), se realizó un barrido sistemático de las placas, deteniéndose en los bordes. Los complejos cúmulo-corona-ovocitos fueron identificados en forma relativamente fácil, porque, en la mayoría de los casos, destacaban por el aspecto refringente del cúmulo que lo rodeaba. Los ovocitos presentes, fueron transferidos a una placa de doble pozo (IVF Culture Dish, 60 × 15 mm, 20260, SPL), colocándose en el compartimiento marginal (contentivo de $1,5 \mathrm{~mL}$ del medio G-MOPSTMPlus, 10130, Vitrolife $\AA$ ) donde se realizó un primer lavado de los mismos. El líquido folicular revisado fue recolectado en un envase recolector de orina estéril para una segunda revisión, siendo posteriormente descartado.

Los ovocitos seleccionados, se transfirieron al compartimiento central de la placa de doble pozo (contentivo de $3 \mathrm{~mL}$ de medio G-MOPSTMPlus, 10130, Vitrolife $\left({ }^{\circledR}\right)$, permaneciendo en la incubadora hasta el momento de la fecundación.

Los datos fueron organizados en una matriz que incluyó, de forma individualizada, todas las variables reportadas por cada ciclo de FIV o ICSI.

Para este estudio, se incluyeron todas las variables implicadas en el Centro en los ciclos de FIV e ICSI antes del aspirado folicular y se consideraron dos 


\section{SÍNDROME DE FOLÍCULO VACÍO EN MUJERES INFÉRTILES SOMETIDAS A TÉCNICAS DE REPRODUCCIÓN ASISTIDA DE ALTA COMPLEJIDAD}

grupos: el grupo de pacientes con SFV y el grupo de pacientes control (sin SFV).

\section{Análisis estadístico:}

De acuerdo con lo anteriormente señalado, se calculó la incidencia y la recurrencia del SFV. Adicionalmente, debido a la importancia de la variable edad, para evaluar su posible dependencia con el SFV, se categorizó en tres grupos: menores de 35 años, entre 35 y 38 años, y mayores de 38 años, aplicándose una tabla de doble entrada o cruzada para evaluar la independencia con la aparición SFV, mediante una prueba de Chi cuadrado.

Luego, para caracterizar las variables continuas: edad, número de folículos periovulatorios, dosis de FSH, LH y antagonista GnRH, así como niveles séricos de hormona anti-mülleriana (HAM), estradiol, LH y FSH de acuerdo con la aparición del SFV, se aplicó una prueba de $t$ de Student para comparación de medias de dos muestras.

Las variables continuas que presentaron diferencias significativas en ambos grupos fueron evaluadas mediante un ANOVA de 1 vía, considerando los tres grupos basados en la edad de las pacientes. Finalmente, se llevó a cabo el mismo procedimiento considerando seis grupos basados en los tres grupos mencionados anteriormente combinados con la aparición del SFV (presencia y ausencia).

Los datos fueron analizados con el programa Statistix for Windows 2008. Versión 8. Analytical Software. Tallahassee, Florida, USA.

\section{RESULTADOS}

La incidencia del SFV fue de 6,18 \%, es decir, se presentaron 23 casos de SFV de un total de 372 ciclos. De las 23 pacientes con SFV, solo 10 se sometieron a más de un estímulo de FIV, previo o siguiente, y solo hubo recurrencia del SFV en dos pacientes, obteniendo un $20 \%$ de recurrencia.

En la tabla 1, se observa como la incidencia del SFV aumenta con la edad de la paciente, siendo significativamente mayor $(11,2 \%)$ en las pacientes mayores de 38 años, comparado con las pacientes menores de 35 años y las pacientes entre 35 y 38 años $(\mathrm{p}<0,05)$.

Los hallazgos de esta investigación muestran que en pacientes menores de 35 años y pacientes entre 35 a 38 años de edad, no hubo recurrencia del SFV; sin embargo, en aquellas pacientes mayores de 38 años existió un 33,3\% de recurrencia del SFV.

La tabla 2 muestra las variables continuas en pacientes de ambos grupos. En cuanto al estímulo ovárico controlado, fue similar en ambos grupos, no encontrándose diferencias significativas $(p>0,05)$ en cuanto a las dosis suministradas de FSH (Control: $2459 \pm 917,6$ UI vs. SFV: $2917 \pm 1121,9$ UI), LH (Control: 230,3 \pm 192,4 UI vs. SFV: $128,1 \pm 167,1$ UI), y antGnRH (Control: $0,9 \pm 0,7 \mathrm{mg}$ vs. SFV: 0,4 $\pm 0,7 \mathrm{mg}$ ).

El promedio de edad de la paciente no mostró diferencia significativa $(\mathrm{p}>0,05)$ entre el grupo control $(34,8 \pm$ 5,1 años) y el grupo de pacientes con SFV $(36,6 \pm 5,7$

Tabla 1. Distribución comparativa de pacientes por grupo de edad

\begin{tabular}{lcc}
\hline \multirow{2}{*}{ EDAD (años) } & Control n $(\%)$ & SFV n $(\%)$ \\
\hline Menos de 35 & $148(97,4)$ & $4(2,6)$ \\
$35-38$ & $106(93,8)$ & $7(6,2)$ \\
Más de 38 & $95(88,8)$ & $12(11,2)$ \\
\hline $\mathrm{p}=0,019$ & &
\end{tabular}


Tabla 2. Distribución comparativa de las variables continuas

\begin{tabular}{lccc}
\hline Variable & Síndrome de folículo vacío & Sin síndrome de folículo vacío & \multirow{2}{*}{$\mathrm{p}$} \\
\cline { 2 - 3 } $\begin{array}{lccc}\text { Dosis suministrada de FSH } \\
\text { (UI) }\end{array}$ & media \pm DS & media \pm DS & $>0,05$ \\
$\begin{array}{l}\text { Dosis suministrada de LH } \\
\text { (UI) }\end{array}$ & $128,1 \pm \pm 1121,9$ & $2459 \pm 917,6$ & $>0,05$ \\
$\begin{array}{l}\text { Dosis suministrada de } \\
\text { antGnRH (mg) }\end{array}$ & $0,4 \pm 0,7$ & $230,3 \pm 192,4$ & $>0,05$ \\
Edad (años) & $36,6 \pm 5,7$ & $0,9 \pm 0,7$ & $>0,05$ \\
No de Folículos & $5,9 \pm 6,4$ & $34,8 \pm 5,1$ & $>0,05$ \\
$\begin{array}{l}\text { Niveles séricos de AMH } \\
\text { (ng/mL) }\end{array}$ & $0,6 \pm 0,3$ & $10,3 \pm 5,3$ & $<0,05$ \\
$\begin{array}{l}\text { Niveles séricos de FSH } \\
\text { basal (ng/mL) }\end{array}$ & $19,1 \pm 13,8$ & $2,1 \pm 2,5$ & $<0,05$ \\
$\begin{array}{l}\text { Niveles séricos de LH } \\
\text { basal (ng/mL) }\end{array}$ & $10,4 \pm 9,8$ & $12,9 \pm 9,0$ & $<0,05$ \\
$\begin{array}{l}\text { Niveles séricos de } \\
\text { estradiol basal (pg/mL) }\end{array}$ & $65,9 \pm 71,4$ & $6,96 \pm 5,3$ & $>0,05$ \\
$\begin{array}{l}\text { Niveles séricos de } \\
\text { estradiol día hCG (pg/mL) }\end{array}$ & $626,6 \pm 571,8$ & $61,2 \pm 54,4$ & $<0,05$ \\
\hline
\end{tabular}

años); la misma relación se encontró al comparar el número de folículos periovulatorios durante el EOC (Control: 10,3 $\pm 5,3$ folículos vs. SFV: 5,9 $\pm 6,4$ folículos; $p>0,05$ ).

Al evaluar los niveles hormonales detectados en suero, se encontraron diferencias significativas entre los grupos. Los niveles de HAM detectados previo al EOC, fueron significativamente más bajos $(p<0,05)$ en aquellas pacientes en las que se presentó el SFV $(0,6 \pm 0,3 \mathrm{ng} / \mathrm{mL})$, respecto al grupo control $(2,1 \pm$ $2,5 \mathrm{ng} / \mathrm{mL}$ ). También, se observó la misma relación al evaluar los niveles de estradiol el día de la inyección de hCG (grupo control: 1573,4 $\pm 1234 \mathrm{pg} / \mathrm{mL}$; grupo SFV: $626,6 \pm 571,8 \mathrm{pg} / \mathrm{mL} ; \mathrm{p}<0,05)$. En cuanto a los niveles basales de FSH y LH, se observó que eran significativamente mayores $(\mathrm{p}<0,05)$ en las pacientes con SFV (FSH: 19,1 $\pm 13,8 \mathrm{ng} / \mathrm{mL}$; LH: 10,4 $\pm 9,8$ $\mathrm{ng} / \mathrm{mL}$ ), respecto al grupo control (FSH: 12,9 $\pm 9,0$ $\mathrm{ng} / \mathrm{mL}$; LH: $6,96 \pm 5,3 \mathrm{ng} / \mathrm{mL}$ ). Por el contrario, los niveles basales de estradiol fueron similares entre el grupo control $(61,2 \pm 54,4 \mathrm{pg} / \mathrm{mL})$ y las pacientes con $\operatorname{SFV}(65,9 \pm 71,4 \mathrm{pg} / \mathrm{mL})$.

La categorización de la variable edad de la paciente (Tabla 3), demostró, que el aumento de los niveles basales de FSH y de LH, así como la disminución de los niveles de estradiol, el día de la inyección hCG, y del número de folículos periovulatorios presentes durante el EOC, están asociados directamente con el aumento de la edad de la paciente $(\mathrm{p}<0,05)$.

En la tabla 4, se observa que se mantienen las mismas diferencias descritas; sin embargo, se aprecia claramente que la presencia del SFV acentúa significativamente las tendencias descritas de las variables en cuestión. Los niveles séricos basales de FSH y LH aumentan significativamente $(p<0,05)$ con la edad de las pacientes que presentan el SFV, respecto a las pacientes sin SFV. Por 


\section{SÍNDROME DE FOLÍCULO VACÍO EN MUJERES INFÉRTILES SOMETIDAS A TÉCNICAS DE REPRODUCCIÓN ASISTIDA DE ALTA COMPLEJIDAD}

Tabla 3. Valores promedios y desviaciones estándar de los niveles séricos de HAM, FSH, LH y estradiol (día de la administración de hCG), y número de folículos periovulatorios, en tres grupos de edades

\begin{tabular}{|c|c|c|c|c|c|c|c|c|c|c|c|c|c|c|c|}
\hline \multirow{2}{*}{$\begin{array}{l}\text { Edad } \\
\text { (años) }\end{array}$} & \multicolumn{3}{|c|}{ HAM } & \multicolumn{3}{|c|}{ FSH } & \multicolumn{3}{|c|}{$\mathrm{LH}$} & \multicolumn{3}{|c|}{ Estradiol } & \multicolumn{3}{|c|}{$\begin{array}{l}\mathrm{N}^{\circ} \text { folículos } \\
\text { periovulatorios }\end{array}$} \\
\hline & $\mathrm{N}$ & Promedio & DS & $\mathrm{N}$ & Promedio & DS & $\mathrm{N}$ & Promedio & DS & $\mathrm{N}$ & Promedio & DS & $\mathrm{N}$ & Promedio & DS \\
\hline$<35$ & 10 & 2,55 & 0,76 & 79 & 10,43 & 1,01 & 79 & 5,63 & 0,62 & 69 & 1590,2 & 143,22 & 101 & 12,16 & 0,5 \\
\hline $35-38$ & 17 & 2,2 & 0,58 & 57 & 13,34 & 1,19 & 56 & 7,95 & 0,74 & 53 & 1787,9 & 163,41 & 74 & 9,78 & 0,59 \\
\hline$>38$ & 15 & 1,2 & 0,62 & 52 & 17,3 & 1,25 & 57 & 8,49 & 0,73 & 38 & 969,6 & 192,99 & 72 & 7,13 & 0,59 \\
\hline & & $\mathrm{p}>0,05$ & & & $\mathrm{p}<0,05$ & & & $\mathrm{p}<0,05$ & & & $\mathrm{p}<0,05$ & & & $\mathrm{p}<0,05$ & \\
\hline
\end{tabular}

otra parte, los niveles séricos de estradiol, el día de la inyección de hCG, y el número de folículos periovulatorios, presentes durante el EOC, descienden significativamente $(p<0,05)$ en las pacientes de edad avanzada con SFV, en relación con las pacientes de edad avanzada sin SFV.

Tabla 4. Valores promedios y desviaciones estándar de los niveles séricos de HAM, FSH, LH y estradiol (día hCG), y número de folículos periovulatorios en seis categorías

\begin{tabular}{|c|c|c|c|c|c|c|c|c|c|c|c|c|c|c|c|}
\hline \multirow{2}{*}{$\begin{array}{l}\text { Edad } \\
\text { (años) }\end{array}$} & \multicolumn{3}{|c|}{ HAM } & \multicolumn{3}{|c|}{ FSH } & \multicolumn{3}{|c|}{ LH } & \multicolumn{3}{|c|}{ Estradiol } & \multicolumn{3}{|c|}{$\begin{array}{c}\mathrm{N}^{\circ} \text { folículos } \\
\text { periovulatorios }\end{array}$} \\
\hline & $\mathrm{N}$ & Promedio & DS & $\mathrm{N}$ & Promedio & DS & $\mathrm{N}$ & Promedio & DS & $\mathrm{N}$ & Promedio & DS & $\mathrm{N}$ & Promedio & DS \\
\hline $\begin{array}{l}<35 \sin \\
\text { SFV }\end{array}$ & 10 & 2,55 & 0,76 & 78 & 10,40 & 1,01 & 78 & 5,59 & 0,62 & 68 & 159,40 & 143,90 & 98 & 12,00 & 0,50 \\
\hline $\begin{array}{l}<35 \text { con } \\
\text { SFV }\end{array}$ & & & & 1 & 13,00 & 8,95 & 1 & 9,00 & 5,48 & 1 & 1238,00 & 1186,30 & 3 & 17,33 & 2,83 \\
\hline $\begin{array}{l}35-38 \\
\text { sin SFV }\end{array}$ & 14 & 2,55 & 0,64 & 54 & 13,49 & 1,22 & 53 & 8,11 & 0,75 & 50 & 1848,80 & 167,80 & 70 & 10,11 & 0,59 \\
\hline $\begin{array}{l}35-38 \\
\text { con SFV }\end{array}$ & 3 & 0,57 & 1,38 & 3 & 10,70 & 5,17 & 3 & 5,17 & 3,16 & 3 & 772,80 & 684,90 & 4 & 3,75 & 2,45 \\
\hline $\begin{array}{l}>38 \sin \\
\text { SFV30 }\end{array}$ & 12 & 1,25 & 0,69 & 46 & 16,39 & 1,32 & 50 & 7,87 & 0,77 & 31 & 1080,90 & 213,10 & 63 & 7,71 & 0,62 \\
\hline \multirow[t]{2}{*}{$\begin{array}{l}>38 \text { con } \\
\text { SFV }\end{array}$} & 3 & 0,60 & 1,38 & 6 & 24,26 & 3,66 & 7 & 12,88 & 2,07 & 7 & 476,60 & 448,40 & 9 & 3,00 & 1,63 \\
\hline & & $\mathrm{p}>0,05$ & & & $\mathrm{p}<0,05$ & & & $\mathrm{p}<0,05$ & & & $\mathrm{p}<0,05$ & & & $\mathrm{p}<0,05$ & \\
\hline
\end{tabular}




\section{DISCUSIÓN}

La incidencia del SFV reportada, coincide con lo descrito por Ravelli y cols. (2) quienes realizaron una revisión de la literatura y determinaron que la incidencia del SFV se presenta en el rango entre $0,045 \%$ y $7 \%$. Esta variación parece estar asociada a la variabilidad de los grupos de pacientes, como es el caso del estudio de Singh y cols. (10), quienes reportan una incidencia global del 2,38 \%; sin embargo, la incidencia asciende a $6,69 \%$ en las pacientes con EOC, utilizando antagonistas de la GnRH; cabe destacar que, en este estudio, todas las pacientes fueron sometidas a esta clase de estímulo.

La asociación entre el aumento de la incidencia del SFV con la edad materna, ha sido propuesta previamente (2, 11-14). Los resultados de este estudio, a pesar de representar grupos homogéneos en cuanto a la edad de la paciente, evidencian esta asociación, ya que muestran un aumento significativo de la incidencia del SFV con el aumento de la edad materna. Ravelli y cols. (2) encontraron que la incidencia del SFV en pacientes mayores de 40 años puede llegar a ser cinco veces más alta que en pacientes menores de 40 años (6,3\% vs $1,8 \%$ ). Incluso, algunos autores proponen la edad, la baja reserva ovárica y pacientes bajas respondedoras como factores de predisposición del SFV genuino (2, 15).

En términos de recurrencia del SFV, se ha reportado en un $15,8 \%$ a $20 \%$ de las pacientes $(12,16)$, lo cual coincide con los resultados de este estudio (20\%). La recurrencia del SFV en pacientes de edad avanzada (> 38 años), también fue reportada por Zreik y cols. (16), quienes concluyen que la recurrencia del SFV aumenta con la edad de la paciente; en ese caso, ninguna de las pacientes $\leq 34$ años, presentó recurrencia del $\mathrm{SFV}$, mientras que, en las pacientes de 35 a 39 años, el porcentaje de recurrencia fue del 23,5\% y en las pacientes $\geq 40$ años, se presentó una recurrencia del $57 \%$.
Cabe destacar que las dos pacientes de este estudio que presentaron recurrencia del SFV, se sometieron a varios ciclos de FIV y en ninguno lograron embarazo; posiblemente, la mejor opción para lograr embarazo en estas pacientes es la donación de ovocitos $(12,17)$; sin embargo, es difícil aseverar esta propuesta, debido a que en estos casos no fue posible la clasificación del SFV, pudiendo haber estado en presencia de SFV falso, causado simplemente por error humano. La recurrencia del SFV o posterior recuperación de ovocitos inmaduros continúa siendo un problema desafiante (18).

Por otra parte, el EOC es el primer paso para la realización de una fecundación in vitro. En el EOC las dosis suministradas de FSH, LH y antGnRH son propuestas por el médico, personalizadas y dependen fundamentalmente de los siguientes criterios: edad de la paciente, el contaje de folículos antrales, niveles hormonales y los antecedentes de la paciente, entre otros (19). Inicialmente, pudieran existir diferencias particulares en cuanto al EOC en las pacientes con SFV; sin embargo, los análisis demuestran que el EOC utilizado en el Centro fue similar en ambos grupos.

Antes de dar inicio al EOC, los niveles de HAM junto con el recuento de folículos antrales, son los mejores predictores de la reserva ovárica, y de la misma dependerá la respuesta de la paciente al estímulo ovárico en la mayoría de los casos. En estas pacientes, los niveles séricos de HAM son reducidos $(<1,26 \mathrm{ng} /$ $\mathrm{mL}$ ) (20). En las pacientes con SFV se han descrito niveles de HAM bajos $(<1 \mathrm{ng} / \mathrm{mL})(14)$, al igual que en la presente investigación $(0,6 \pm 0,3 \mathrm{ng} / \mathrm{mL})$.

Durante el EOC los niveles de estradiol se cuantifican para monitorear el desarrollo folicular, con el fin de obtener un número adecuado de ovocitos maduros y prevenir el riesgo de hiperestimulación ovárica (21), siendo más relevantes los niveles basales y la medición el día de la inyección de hCG. Los niveles de estradiol se han asociado con una alta tasa de embarazo. Los 


\section{SÍNDROME DE FOLÍCULO VACÍO EN MUJERES INFÉRTILES SOMETIDAS A TÉCNICAS DE REPRODUCCIÓN ASISTIDA DE ALTA COMPLEJIDAD}

niveles basales de esta hormona, deben superar los $31,2 \mathrm{pg} / \mathrm{mL}$ y el día la inyección de hCG deben ser $>1400 \mathrm{pg} / \mathrm{mL}$ (22). Por el contrario, los niveles de estradiol el día de la inyección hCG menores a 500 $\mathrm{pg} / \mathrm{mL}$, se han relacionado significativamente con una baja reserva ovárica (23). En el grupo de pacientes con SFV, los niveles de estradiol el día de la administración de hCG fueron significativamente menores (grupo control: 1573,4 $\pm 1234 \mathrm{pg} / \mathrm{mL}$; grupo SFV: $626,6 \pm$ $571,8 \mathrm{pg} / \mathrm{mL} ; \mathrm{p}<0,05)$, estos resultados concuerdan con lo reportado por Baum y cols. (12) (1516,3 \pm $887,5 \mathrm{pg} / \mathrm{mL}$ vs. $499,9 \pm 480,9 \mathrm{pg} / \mathrm{mL}, \mathrm{p}<0,001)$, Hasegawa y cols. (13) (1717 pg/mLvs. 633 pg/mL, p $<0,001)$, Singh y cols (10) $(4745 \mathrm{pg} / \mathrm{mL}$ vs. $939 \mathrm{pg} /$ $\mathrm{mL}, \mathrm{p}<0,01)$ y Zreik y cols. (16) $(869,1 \mathrm{pg} / \mathrm{mL}$ vs. $641,6 \mathrm{pg} / \mathrm{mL}, \mathrm{p}<0,001)$.

Asimismo, la aparición del SFV está asociada a un incremento en los niveles séricos basales de FSH y LH y a una disminución en el número de folículos periovulatorios (12 - 14), dicha aseveración es corroborada en esta investigación (FSH: 19,1 $\pm 13,8$ $\mathrm{ng} / \mathrm{mL}$ vs. $12,9 \pm 9,0 \mathrm{ng} / \mathrm{mL}$; LH: $10,4 \pm 9,8 \mathrm{ng} / \mathrm{mL}$ vs. $6,96 \pm 5,3 \mathrm{ng} / \mathrm{mL} ; \mathrm{N}^{\circ}$ folículos periovulatorios: $5,9 \pm$ $6,4$ vs. $10,3 \pm 5,3 ; \mathrm{p}<0,05)$.

Los resultados descritos, han sido asociados a baja reserva ovárica y a pacientes bajas respondedoras (20, $23,24)$, aunque vale la pena resaltar el poco consenso que existía hasta hace pocos años en cuanto a ambas definiciones, encontrándose en la literatura múltiples definiciones de pacientes bajas respondedoras. El criterio de Bologna, en 2011, estandarizó la definición (25), estipulando que una paciente es considerada baja respondedora, si cumple con dos de las tres características siguientes: edad materna avanzada (mayor de 40 años) o cualquier otro factor de riesgo de baja respuesta, ciclo previo con baja respuesta (menos de tres ovocitos recuperados) y alteración de la prueba de reserva ovárica (recuento de folículos antrales entre 5 y 7 o HAM 0,5-1,1 ng/mL); sin embargo, siendo parcialmente exitoso, la definición permanecía controversial y continuaba siendo un problema complejo, ya que en los ensayos clínicos se corría el riesgo de agrupar pacientes que diferían significativamente en sus características biológicas. En 2016, el grupo POSEIDON (Patient-Oriented Strategies Encompassing Individualized Oocyte Number) introdujo una definición más detallada de las pacientes con bajo pronóstico con una nueva estratificación, identificando grupos de pacientes con pronóstico potencialmente diferentes, e incluyó una guía para mejorar el manejo de las pacientes con este problema que se someten a técnicas de reproducción asistida (26).

En los casos de FIV en pacientes con baja respuesta ovárica, se ha asociado un mal pronóstico, siendo vulnerables a fallas en la recuperación de ovocitos, incluso, en la mayoría de las pacientes, los ciclos de FIV se cancelan antes de la recuperación de ovocitos y aproximadamente el $30 \%$ de las pacientes no logra la transferencia de embriones, ya sea por una aspiración folicular sin ovocitos recuperados o por la baja cantidad de ovocitos recuperados $(20,24,27)$.

En virtud de minimizar la posibilidad de la no recuperación de ovocitos por baja respuesta ovárica, por problemas técnicos durante la aspiración folicular (ante un número reducido de folículos) o sencillamente por las bajas probabilidades de recuperación de ovocitos (28), se ha considerado como criterio, en estudios del SFV, la exclusión de pacientes con baja reserva ovárica y bajas respondedoras reportando tasas de incidencia tan bajas como 0,045 \% $(3,4,29)$.

En este estudio, es evidente que gran parte del grupo de pacientes con SFV están asociadas a baja respuesta ante el EOC, considerando el criterio de POSEIDON (26). Para actualizar y simplificar los datos, un $69 \%$ de las pacientes con SFV correspondería al grupo 4, pacientes mayores de 35 años de edad con parámetros previos a la estimulación de baja reserva ovárica 
(recuento de folículos antrales $<5$ o HAM $<1,2 \mathrm{ng}$ / $\mathrm{mL})$, la exclusión de este grupo de pacientes reduciría la incidencia a un $1,88 \%$ y la recurrencia del SFV no se habría presentado.

Sin embargo, otros autores consideran que estas pacientes deben ser incluidas y su exclusión subestima la incidencia del SFV (2) o deben ser evaluadas aparte (4). En este sentido, Verberg y cols. (30) indican que las estimulaciones ováricas leves, que reducen los costos y las molestias a las pacientes, no reducen las tasas de embarazo, ni las tasas de recuperación de ovocitos; por lo que no es justificado la exclusión de estas pacientes.

Como bien es sabido, es un hecho que la reserva ovárica disminuye a medida que aumenta la edad de la mujer debido al envejecimiento ovárico, lo cual estaría asociado a una baja respuesta ante el EOC. En este estudio, se corrobora este enunciado, al discriminar las pacientes en grupos etarios. Sin embargo, es interesante resaltar, como se acentúa esta asociación ante la presencia del SFV, presentando diferencia significativa entre los grupos.

Lamentablemente, una de las limitaciones del estudio fue la imposibilidad de clasificar los casos de SFV en verdadero o falso (5), debido a que, para llevar a cabo dicha clasificación, se debe implementar la cuantificación los niveles de hCG en suero de la paciente el día de la aspiración de los folículos $(31,32)$ o 12 horas después de la inyección de hCG (29). La predicción y clasificación del SFV, debe considerarse a futuro y se debe evaluar la relación costo beneficio, ya que su detección permitiría conocer si la aparición del SFV es causada simplemente por error humano o, por el contrario, facilitaría información valiosa para el entendimiento del SFV.

Adicionalmente, se recomienda considerar en pacientes con SFV protocolos de rescate que permitan la recuperación del ciclo; la implementación de estos protocolos ha permitido la transferencia de embriones de buena calidad y tasas de embarazo hasta de un $50 \%$ (33). El SFV es una realidad y su manejo debe establecerse.

\section{AGRADECIMIENTOS}

A todo el personal del Centro de Fertilidad CenFer, María Mercedes Ramírez, Thais Díaz, Randolfo Medina y Pedro Cabrera.

\section{REFERENCIAS}

1. Coulam CB, Bustillo M, Schulman JD. Empty follicle syndrome. Fertil Steril. 1986; 46(6):1153-1155. doi: 10.1016/s0015-0282(16)49898-5.

2. Revelli A, Carosso A, Grassi G, Gennarelli G, Canosa S, Benedetto C. Empty follicle syndrome revisited: definition, incidence, aetiology, early diagnosis and treatment. Reprod Biomed Online. 2017; 35(2):132138. doi: 10.1016/j.rbmo.2017.04.012.

3. Kim JH, Jee BC. Empty follicle syndrome. Clin Exp Reprod Med. 2012; 39(4):132-137. doi: 10.5653/ cerm.2012.39.4.132.

4. Mesen TB, Yu B, Richter KS, Widra E, DeCherney AH, Segars JH. The prevalence of genuine empty follicle syndrome. Fertil Steril. 2011; 96(6):1375-7. doi: 10.1016/j.fertnstert.2011.09.047.

5. Stevenson TL, Lashen H. Empty follicle syndrome: the reality of a controversial syndrome, a systematic review. Fertil Steril. 2008; 90(3):691-8. doi: 10.1016/j. fertnstert.2007.07.1312.

6. Rubio J, García Y, Fortuño S, Salas B, Vañó B, Monzó $\mathrm{A}$, et al. Estudio retrospectivo de la ausencia de ovocitos en la punción folicular tras la estimulación ovárica para fecundación in vitro. Revista Fertilidad [Internet]. 2008 [consultado 14 de junio de 2020]; 25(3):169174. Disponible en: http://www.revistafertilidad.org/ RecursosWEB/fertilidad/Mayo-Junio\%202008\%20 trabajo\%204.pdf

7. Gónzalez I, Romero B, Martinez L, Mozas J. Síndrome del folículo vacío genuino. A propósito de un caso. Clín investig ginecol obstet (Ed. impr.). 2016; 43(4):179182.

8. Deepika K, Rathore S, Garg N, Rao K. Empty follicle syndrome: Successful pregnancy following dual trigger. J Hum Reprod Sci. 2015; 8(3):170-174. doi: 10.4103/0974-1208.165152. 


\section{SÍNDROME DE FOLÍCULO VACÍO EN MUJERES INFÉRTILES SOMETIDAS A TÉCNICAS DE REPRODUCCIÓN ASISTIDA DE ALTA COMPLEJIDAD}

9. Blazquez A, Guillén JJ, Colomé C, Coll O, Vassena R, Vernaeve V. Empty follicle syndrome prevalence and management in oocyte donors. Hum Reprod. 2014; 29(10):2221-2227. doi: 10.1093/humrep/deu203.

10. Singh N, Dalal V, Kriplani A, Malhotra N, Mahey R, Perumal V. Empty Follicle Syndrome: A Challenge to Physician. J Hum Reprod Sci. 2018; 11(3):274-278. doi: 10.4103/jhrs.JHRS_61_17.

11. Lorusso F, Depalo R, Tsadilas S, Caradonna F, Di Gilio A, Capotorto MT, et al. Is the occurrence of the empty follicle syndrome a predictor that a subsequent stimulated cycle will be an unfavourable one? Reprod Biomed Online. 2005; 10(5):571-574. doi: 10.1016/ s1472-6483(10)61662-8.

12. Baum M, Machtinger R, Yerushalmi GM, Maman E, Seidman DS, Dor J, et al. Recurrence of empty follicle syndrome with stimulated IVF cycles. Gynecol Endocrinol. 2012; 28(4):293-295. doi: 10.3109/09513590.2011.631629.

13. Hasegawa A, Takahashi T, Igarashi H, Amita M, Matsukawa J, Nagase S. Predictive factors for oocyte retrieval failure in controlled ovarian hyperstimulation protocols: a retrospective observational cohort study. Reprod Biol Endocrinol. 2015; 13:53. doi: 10.1186/ s12958-015-0052-x.

14. Madani T, Jahangiri N. Empty Follicle Syndrome: The Possible Cause of Occurrence. Oman Med J. 2015; 30(6):417-420. doi: 10.5001/omj.2015.83.

15. Yakovi S, Izhaki I, Ben-Ami M, Younis JS. Does the empty follicle syndrome occur in cases of low number of maturing follicles in assisted reproduction? Gynecol Endocrinol. 2019; 35(4):305-308. doi: 10.1080/09513590.2018.1519793.

16. Zreik TG, Garcia-Velasco JA, Vergara TM, Arici A, Olive D, Jones EE. Empty follicle syndrome: evidence for recurrence. Hum Reprod. 2000; 15(5):999-1002. doi: 10.1093/humrep/15.5.999.

17. Kaluarachchi A, Seneviratne HR, Batcha TM, Wijeratne S, Jayawardena GRMUGP. Recurrent empty follicle syndrome with different stimulation protocols - A case report. J Family Med Prim Care. 2019; 8(7):2536-2537. doi: $10.4103 / j f m p c . j f m p c \_418 \_19$.

18. Al-Hussaini TK, Yosef $\bar{A} H, \bar{E}$ l-Nashar IH, Shaaban OM. Repeated recovery of immature oocytes in a woman with a previous history of empty follicle syndrome. JBRA Assist Reprod. 2019; 23(1):72-74. doi: 10.5935/1518-0557.20180068.

19. Cohen J. A short review of ovarian stimulation in assisted reproductive techniques. Reprod Biomed
Online. 2003; 6(3):361-366. doi: 10.1016/s14726483(10)61858-5.

20. Barad DH, Weghofer A, Gleicher N. Utility of agespecific serum anti-Müllerian hormone concentrations. Reprod Biomed Online. 2011; 22(3):284-291. doi: 10.1016/j.rbmo.2010.12.002.

21. Papageorgiou T, Guibert J, Goffinet F, Patrat C, Fulla Y, Janssens Y, et al. Percentile curves of serum estradiol levels during controlled ovarian stimulation in 905 cycles stimulated with recombinant FSH show that high estradiol is not detrimental to IVF outcome. Hum Reprod. 2002; 17(11):2846-2850. doi: 10.1093/ humrep/17.11.2846.

22. Prasad S, Kumar Y, Singhal M, Sharma S. Estradiol Level on Day 2 and Day of Trigger: A Potential Predictor of the IVF-ET Success. J Obstet Gynaecol India. 2014; 64(3):202-207. doi: 10.1007/s13224-0140515-6.

23. Sharara FI, McClamrock HD. High estradiol levels and high oocyte yield are not detrimental to in vitro fertilization outcome. Fertil Steril. 1999; 72(3):401-405. doi: https://doi.org/10.1016/S0015-0282(99)00293-9

24. Gleicher N, Weghofer A, Barad DH. Discordances between follicle stimulating hormone (FSH) and antiMüllerian hormone (AMH) in female infertility. Reprod Biol Endocrinol. 2010; 8:64. doi: 10.1186/1477-78278-64.

25. Ferraretti AP, La Marca A, Fauser BC, Tarlatzis B, Nargund G, Gianaroli L; ESHRE working group on Poor Ovarian Response Definition. ESHRE consensus on the definition of 'poor response' to ovarian stimulation for in vitro fertilization: the Bologna criteria. Hum Reprod. 2011; 26(7):1616-1624. doi: 10.1093/humrep/der092.

26. Poseidon Group (Patient-Oriented Strategies Encompassing IndividualizeD Oocyte Number), Alviggi C, Andersen CY, Buehler K, Conforti A, De Placido G, Esteves SC, et al. A new more detailed stratification of low responders to ovarian stimulation: from a poor ovarian response to a low prognosis concept. Fertil Steril. 2016; 105(6):1452-1453. doi: 10.1016/j.fertnstert.2016.02.005.

27. Gleicher N, Darmon SK, Kushnir VA, Weghofer A, Wang Q, Zhang L, et al. How FSH and AMH reflect probabilities of oocyte numbers in poor prognosis patients with small oocyte yields. Endocrine. 2016; 54(2): 476-483. doi: 10.1007/s12020-016-1068-5.

28. van Heusden AM, van Santbrink EJ, Schipper I, de Jong D. The empty follicle syndrome is dead! Fertil Steril. 2008; 89(3):746. doi: 10.1016/j.fertnstert.2007.12.048. 
29. Reichman DE, Hornstein MD, Jackson KV, Racowsky C. Empty follicle syndrome--does repeat administration of hCG really work? Fertil Steril. 2010; 94(1):375-7. doi: 10.1016/j.fertnstert.2009.09.040.

30. Verberg MF, Eijkemans MJ, Macklon NS, Heijnen EM, Baart EB, Hohmann FP, et al. The clinical significance of the retrieval of a low number of oocytes following mild ovarian stimulation for IVF: a meta-analysis. Hum Reprod Update. 2009; 15(1):5-12. doi: 10.1093/ humupd/dmn053.

31. Ndukwe G, Thornton S, Fishel S, Dowell K, al-Hassan S, Hunter A. Predicting empty follicle syndrome. Fertil Steril. 1996; 66(5):845-847. doi: https://doi. org/10.1016/S0015-0282(16)58650-6
32. Kourtis A, Rousso D, Panidis D. The empty follicle syndrome. J Endocrinol Invest. 2004; 27(2):187-191. doi: 10.1007/BF03346267.

33. Deepika K, Sindhuma D, Kiran B, Ravishankar N, Gautham P, Kamini R. Empty Follicle Syndrome Following GnRHa Trigger in PCOS Patients Undergoing IVF Cycles. J Reprod Infertil [Internet]. 2018 [consultado 14 de junio de 2020]; 19(1):16-25. Disponible en: https://www.ncbi.nlm.nih.gov/pmc/ articles/PMC5960047/

Recibido 20 de agosto de 2020 Aprobado 18 de noviembre de 2020 